\title{
Estrutura fatorial e propriedades psicométricas do Inventário da Tríade Cognitiva
}

\author{
Maycoln Leôni Martins Teodoro' \\ Priscilla Moreira Ohno \\ Mariana Verdolin Guilherme Froeseler \\ Universidade Federal de Minas Gerais, Belo Horizonte - MG - Brasil
}

\begin{abstract}
Resumo: $O$ conceito de tríade cognitiva negativa refere-se à visão disfuncional que o indivíduo possui dele mesmo (self), dos outros e do futuro. O objetivo deste estudo foi investigar a estrutura fatorial e propriedades psicométricas do Inventário da Tríade Cognitiva (ITC). Dos 404 participantes com idade entre 17 e 50 anos (média $=25$, I4; $\mathrm{DP}=8,53$ anos), 280 eram mulheres $(69,3 \%)$. Foram realizadas análises fatoriais confirmatórias utilizando como referência os quatro modelos encontrados na literatura. A partir da comparação dos índices de ajustamento, a solução de seis fatores mostrou-se a mais apropriada, sendo essa formada pela tríade cognitiva negativa original, associada às visões positivas do self, dos outros e do futuro. Foram encontrados índices aceitáveis de consistência interna $(0,61<\alpha>0,75)$, além de correlações significativas entre fatores do ITC e o sexo e a idade dos participantes. Os resultados apontam para a adequação das propriedades psicométricas do ITC nessa amostra.
\end{abstract}

Palavras-chave: terapia cognitiva; escalas; crença cognitiva; propriedades psicométricas; avaliação psicológica.

\section{FACTORIAL STRUCTURE AND PSYCHOMETRIC PROPERTIES OF THE COGNITIVE TRIAD INVENTORY}

\begin{abstract}
The concept of negative cognitive triad refers to the dysfunctional view that the individual owns about himself (self), others and the future. The purpose of this study was to investigate the factor structure and psychometric properties of the Cognitive Triad Inventory (ITC). Of the 404 participants aged between of 17 and 50 years old (average $=25,14$; SD $=8,53$ years), 280 were women $(69,3 \%)$. Confirmatory factor analysis were performed using as reference the four models found in literature. By comparing the adjustment index, the six factor solution were the most appropriate, which contained the original negative cognitive triad, associated with other positive views of the self, others and the future. Acceptable internal consistency index $(0,61<$ $\alpha>0,75$ ), besides significant correlations between the ITC factors and the sex and age of participants. The results point to the adequacy of the psychometric properties of ITC in this sample.
\end{abstract}

Keywords: cognitive therapy; scales; cognitive belief; psychometric properties; psychological assessment.

1 Endereço para correspondência: Maycoln Leôni Martins Teodoro, Avenida Antônio Carlos, 6627, Pampulha, Belo Horizonte - MG - Brasil. CEP: 31270-901. E-mail: mlmteodoro@hotmail.com.

${ }^{2}$ Com o apoio do Conselho Nacional de Desenvolvimento Científico e Tecnológico (CNPq) e Fundação de Amparo à Pesquisa do Estado de Minas Gerais (Fapemig). 
FACTORIALES ESTRUCTURA Y PROPIEDADES PSICOMÉTRICAS DE INVENTARIO TRÍADE COGNITIVA

\begin{abstract}
Resumen: El concepto de la tríade cognitiva negativa se refiere a la vista disfuncional que el individuo tiene de sí mismo (self), de los demás y del futuro. El objetivo de este estudio fue investigar la estructura factorial y propiedades psicométricas del Inventario Tríada Cognitiva (ITC). De los 404 participantes de edades comprendidas entre 17 y 50 años $(\mathrm{SD}=8,53$ años media $=25,14), 280$ eran mujeres $(69,3 \%)$. Fueran emprendidas análisis factoriales confirmatorias utilizando los cuatro modelos encontrados en la literatura como referencia. De la comparación entre los índices de ajuste, la solución de seis factores resulto la más adecuada, conteniendo la tríade cognitiva negativa original, asociada con una visión positiva de sí mismo, los demás y el futuro. Fueran encontrados niveles aceptables de consistencia interna $(0,61<\alpha>0,75)$, $y$ correlaciones significativas entre los factores del ITC y la edad y sexo de los participantes. Los resultados indican la adecuación de las propiedades psicométricas de la ITC en esta.
\end{abstract}

Palabras clave: terapia cognitiva; escalas; crencia cognitiva; propiedades psicométricas; evaluación psicológica.

O modelo cognitivo desenvolvido por Beck trabalha com a hipótese de que as reações emocionais e comportamentais das pessoas diante das situações estão diretamente relacionadas à maneira com a qual elas percebem e processam os eventos (Beck, Rush, Shaw, \& Emery, 1997; Beck \& Alford, 2011). Assim, mais do que a situação em si, são as interpretações das situações que influenciam os sentimentos e comportamentos. Além disso, acredita-se que haja uma relação recíproca entre a cognição e o afeto, na qual um reforçaria o outro, possibilitando um aumento da limitação cognitiva e emocional (Dobson \& Dozois, 2006). Fundamental para o entendimento desse processo é o conceito desenvolvido por Beck de crenças centrais (Beck et al., 1997).

As crenças centrais são ideias profundas, globais e rígidas que o indivíduo possui sobre si mesmo, sobre os outros e sobre seu futuro (Beck et al., 1997). Elas são desenvolvidas desde cedo na infância, a partir de vivências iniciais e dos relacionamentos com figuras significativas, e são tidas como verdades absolutas e inquestionáveis pelo sujeito. As crenças centrais atuam como lentes que, quando ativadas, aumentam o foco do indivíduo para as evidências que as comprovem, desconsiderando as informações contrárias à interpretação e levando à sua manutenção. Quando bem ajustadas, as crenças possibilitam interpretações funcionais das situações. Contudo, se ativadas em contextos incompatíveis, as mesmas crenças podem levar o indivíduo a conclusões distorcidas e desadaptadas (Neufeld \& Cavenage, 2010). As crenças negativas são frequentes em psicopatologias, como a depressão. Elas exercem um papel importante no desenvolvimento e na manutenção dos sintomas depressivos (Pössel \& Knopf, 2008).

Beck aponta que as crenças cognitivas podem ser analisadas a partir de três perspectivas, que ele denominou tríade cognitiva (Beck et al., 1997). A tríade cognitiva consiste em padrões cognitivos por meio dos quais os indivíduos pensam e interpretam os fatos em relação a si mesmos, aos outros/mundo e ao futuro. Em pacientes deprimidos, essa tríade se configura de maneira negativa. O primeiro componente da tríade é a percepção negativa que a pessoa deprimida tem de si mesma, como alguém 
indesejável, inadequado e sem valor, tendendo a atribuir a culpa por experiências desagradáveis a si mesmo, a seus defeitos mentais, morais ou físicos. O segundo componente corresponde à tendência a interpretar a realidade como ameaçadora, exigente e cheia de obstáculos insuperáveis. Geralmente, as interações da pessoa deprimida com o meio são representativas de sua derrota ou depreciação, e ela tende a ver sua vida repleta de obstáculos ou situações traumáticas. O terceiro componente é a visão negativa da pessoa deprimida em relação ao futuro, do qual ela espera a permanência das privações, dificuldades, frustrações e fracassos, e que o seu sofrimento continuará indefinidamente (Beck et al., 1997; Beck \& Alford, 2011).

Estudos empíricos conduzidos com diferentes faixas etárias têm indicado importantes associações entre a tríade cognitiva e sintomas depressivos (Esbense \& Benson, 2007; Greening, Stoppelbein, Dhossche, \& Martin, 2005; Haaga, Dyck, \& Ernst, 1991; Jacobs \& Joseph, 1997; Pössel, 2009). Nesse sentido, a função da tríade, dentro do modelo cognitivo, seria de ativar outros sintomas negativos e colaborar para os sintomas depressivos na medida em que influencia erroneamente a interpretação do paciente de que está sendo rejeitado.

Diante do importante papel da tríade cognitiva no modelo cognitivo da depressão, Beckham, Leber, Wastkins, Boyer e Cook (1986) desenvolveram o primeiro instrumento direcionado à sua avaliação. O processo de geração de itens do Cognitive Triad Inventory (CTI) iniciou-se com a criação de 36 itens relacionados aos fatores teóricos das visões negativas dos indivíduos sobre si mesmos, sobre os outros e sobre o futuro, compondo a tríade cognitiva originalmente desenvolvida por Beck. Em seguida, os itens foram aplicados em 26 pacientes deprimidos, sendo analisadas as correlações de cada sentença com o fator de origem. Os autores analisaram ainda a correlação do CTI com os inventários de desesperança de Beck e com a escala de autoestima de Rosemberg em outra amostra (28 pacientes). A versão final do CTI apresentou índices de consistência interna de 0,95 para a escala total, 0,91 para visão de self, 0,81 para visão de mundo, 0,93 para visão de futuro. Foram encontradas correlações significativas e positivas entre o CTI e o Inventário de Depressão de Beck (BDI), além de evidências de validade convergente e divergente com os demais instrumentos. Apesar de considerar a tríade cognitiva negativa na construção do inventário, os autores não investigaram empiricamente sua estrutura neste estudo.

Anderson e Skidmore (1995) investigaram o CTI em uma amostra não clínica de 260 estudantes universitários. O modelo teórico de três fatores não apresentou resultados satisfatórios, com uma distribuição de itens que não representava a estrutura original. A partir desses resultados, foi hipotetizado um modelo com seis fatores, que abrigassem construtos positivos e negativos da tríade. Para testar esse modelo foi realizada uma análise para seis fatores, mas somente cinco conseguiram um autovalor superior a 1. Os cinco fatores representavam agrupamentos de itens positivos do futuro, do mundo e do self e itens negativos do futuro e do mundo. Os itens negativos do self se distribuíram nesses cinco fatores. 
Em outro estudo, McIntosh e Fisher (2000) testaram o CTI em uma amostra de 641 estudantes universitários. Ao conduzirem uma análise fatorial confirmatória para o modelo trifatorial de Beck, os autores encontraram altos índices de correlação entre fatores, indicando que uma única dimensão subjacente poderia estar presente. Posteriormente, os autores realizaram uma análise fatorial exploratória confirmando a solução de um único fator para o CTI, nomeado pelos autores de "Atitude Autonegativa Relevante".

Em um estudo realizado na Alemanha, Pössel (2009) investigou 796 estudantes, funcionários e corpo docente de uma universidade para validar a versão alemã do CTI, testando a qualidade dos itens e a estrutura dos fatores presentes nesta tradução. A análise dos itens da versão revelou propriedades psicométricas semelhantes à versão original do CTI (Beckham et al., 1986). Com o intuito de verificar qual o modelo fatorial se ajustava melhor aos dados, o autor realizou análises fatoriais confirmatórias dos modelos de um (Mclntosh \& Fisher, 2000), três (Beckham et al., 1986) e cinco fatores (Anderson \& Skidmore, 1995). Junto a esses modelos foi testado outro de seis fatores, sugerido por Anderson e Skidmore (1995), diante dos resultados obtidos em seu estudo. A partir de comparações entre os índices de ajustamento dos diferentes modelos, Pössel (2009) verificou que o modelo de seis fatores era o que melhor se ajustava aos dados. Os resultados apontaram para a validade da separação da tríade cognitiva em três elementos distintos, porém, associados, e para a existência de estruturas cognitivas equivalentes entre indivíduos norte-americanos e alemães, sugerindo a possível generalização do modelo de Beck em outras culturas. Além disso, a divisão dos itens em fatores positivos e negativos pode não ser um simples reflexo da formulação dos itens, e sim uma evidência da independência entre esses elementos e de suas contribuições para o bem-estar psicológico.

Na Polônia, Śliwerski (2014) avaliou 29 pacientes deprimidos, 29 prisioneiros e 28 estudantes universitários, a fim de investigar as propriedades psicométricas preliminares da versão polonesa do CTI. No que diz respeito à consistência interna do instrumento, todos os itens tiveram uma correlação item-fator igual ou superior a 0,46 com sua escala original, alphas de Cronbach variando de 0,77 a 0,91 nas subescalas e de 0,95 para a escala total, e correlações teste-reteste com um intervalo de quatro semanas entre 0,78 e 0,83. A análise fatorial exploratória indicou a presença de sete fatores com autovalores acima de 1,00. No entanto, o gráfico de sedimentação apontou para uma estrutura bidimensional que explicaria $42,79 \%$ da variância. Ao analisar os dois fatores o autor percebeu que os itens relacionados ao self e futuro se agruparam em um único fator e os itens sobre o mundo se mantiveram em um fator isolado com cargas fatoriais não satisfatórias. Diante disso, a solução unifatorial foi adotada como a que melhor se ajustava aos dados.

Considerando a importância da tríade cognitiva no estudo da depressão e a ausência de instrumentos que possibilitem sua avaliação no contexto brasileiro, torna-se premente a adaptação do Cognitive Triad Inventory (Beckham, Leber, Wastkins, Boyer, \& 
Cook, 1986) para nossa realidade. Nesse sentido, o presente estudo objetiva avaliar a estrutura fatorial e algumas propriedades psicométricas (validade de construto e fidedignidade) da adaptação da versão em português desse instrumento.

\section{Método}

\section{Participantes}

Participaram deste estudo 404 adolescentes e adultos oriundos de grupos universitários, alunos do $1^{\circ}$ período do curso de Psicologia da Universidade Federal de Minas Gerais (68\%), de pais de alunos de escolas públicas $(22,8 \%)$ e de integrantes de grupos artísticos (9,2\%). A idade variou de 17 a 50 anos (média =25,14; DP = 8,53 anos), sendo 280 mulheres $(69,3 \%)$ e 123 homens (30,4\%). Um participante não informou o sexo $(0,2 \%)$. O critério de seleção amostral foi o de conveniência.

\section{Instrumento}

O Cognitive Triad Inventory (Inventário da Tríade Cognitiva [ITC] - Beckham, Leber, Wastkins, Boyer, \& Cook, 1986) investiga pensamentos relativos à tríade cognitiva de Beck (Beck et al., 1997). Os três fatores teóricos que compõem a escala são formados por ideias envolvendo a visão de si mesmo (self) (por exemplo, "Eu posso fazer bem várias coisas"), do mundo (por exemplo, "Não importa o que eu faça, as outras pessoas tornam as coisas que eu quero mais difíceis") e do futuro (por exemplo, "Espero estar contente e satisfeito com o passar dos anos"). O instrumento possui 36 itens, sendo seis frases de preenchimento que não pertencem a nenhum dos três fatores propostos originalmente. O instrumento é respondido por meio de uma escala Likert de sete pontos, variando de 1 (concorda totalmente) a 7 (discorda totalmente).

A tradução do Cognitive Triad Inventory para o português foi realizada por três tradutores de maneira independente. As traduções foram discutidas por um comitê formado por juízes que formularam uma versão inicial do Inventário da Tríade Cognitiva (ITC). Essa versão foi novamente traduzida para o inglês e comparada com a original, apontando a compatibilidade entre as versões e a ausência da necessidade de alterações na formulação dos itens, tendo como resultado a versão final do ITC.

\section{Procedimentos de pesquisa}

A aplicação do ITC aos participantes foi feita de maneira coletiva, com exceção dos pais dos alunos de escolas públicas que receberam o instrumento e o Termo de Consentimento Livre e Esclarecido por intermédio de seus filhos em um envelope lacrado. Ambos (ITC e o TCLE) foram devolvidos aos pesquisadores na mesma condição de sigilo, cabendo destacar que somente foram incluídos nas análises os instrumentos acompanhados de termos assinados. Depois do contato inicial com os adolescentes e adultos, foi informado o objetivo geral do estudo e aqueles interessados assinaram o Termo de Consentimento Livre e Esclarecido; no caso dos adolescentes, a autorização 
foi dada por seus responsáveis. O projeto de pesquisa foi aprovado pelo comitê de ética da Universidade Vale dos Sinos (Unisinos) (Protocolo n. 0041.0.390.000-09).

\section{Análise dos dados}

A investigação das propriedades psicométricas do ITC foi realizada por meio de análise fatorial confirmatória (AFC), de consistência interna (Alpha de Cronbach) e correlações item-fator. Na primeira (AFC), foram comparados os quatro modelos encontrados na literatura internacional (um, três, cinco e seis fatores), a fim de identificar o que melhor se ajustava à adaptação para o português. Os modelos foram analisados por meio da razão do valor do Qui-Quadrado com o grau de liberdade (valores inferiores a cinco foram considerados aceitáveis), do Root Mean Squared of the Residuals (RMSEA, valores abaixo de 0,08 considerados como aceitáveis), do Comparative Fit Index (CFI) e do The Tucker-Lewis Index (Non-Normed Fit Index) (NNFI). Para ambos CFI e NNFI, escores superiores a 0,90 foram considerados satisfatórios. A comparação entre os modelos foi feita pelo Akaike Information Criterion (AIC), na qual o menor valor indicou o meIhor modelo (Akaike, 1974). As comparações entre grupos foram realizadas com o Teste $t$ e as associações foram feitas por meio de correlação de Pearson. Toda a análise estatística foi realizada nos programas estatísticos Lisrel 8.8 e SPSS 16.

\section{Resultados}

\section{Teste dos modelos (AFC e Alphas de Cronbach)}

A análise da estrutura fatorial do ITC foi feita por meio de quatro análises confirmatórias para um, três, cinco e seis fatores, respectivamente. O resumo das análises está descrito na Tabela 1.

Tabela I. Resumo dos resultados para os modelos de um, três, cinco e seis fatores do Inventário da Tríade Cognitiva

\begin{tabular}{llllllll}
\hline Modelo & $\mathrm{X}^{2}$ & $\mathrm{GL}$ & $\mathrm{X}^{2} / \mathrm{GL}$ & $\mathrm{RMSEA}$ & $\mathrm{CFI}$ & $\mathrm{NNFI}$ & $\mathrm{AIC}$ \\
\hline I Fator & $1806, \mathrm{II}$ & 405 & 4,45 & 0,09 & $\mathbf{0 , 8 8}$ & 0,87 & $1921, \mathrm{II}$ \\
\hline 3 Fatores & 1452,78 & 402 & 3,61 & $\mathbf{0 , 0 8}$ & $\mathbf{0 , 9 1}$ & 0,87 & 1578,78 \\
\hline 5 Fatores & 1056,06 & 395 & 2,67 & $\mathbf{0 , 0 6}$ & $\mathbf{0 , 9 3}$ & $\mathbf{0 , 9 3}$ & 1196,06 \\
\hline 6 Fatores & 922,67 & 390 & 2,36 & $\mathbf{0 , 0 5}$ & $\mathbf{0 , 9 4}$ & $\mathbf{0 , 9 3}$ & 1072,67 \\
\hline
\end{tabular}

Nota. $X^{2}=$ Valor do Qui-Quadrado; GL = Grau de Liberdade; RMSEA = Root Mean Squared of the Residuals; CFI = Comparative Fit Index; NNFI = The Tucker-Lewis Index (Non-Normed Fit Index); AIC = Akaike Information Criterion. Escores em negrito indicam índices adequados para o modelo.

Fonte: Elaborada pelos autores. 
O modelo unifatorial, descrito por Mclntosh e Fisher (2000), foi elaborado de modo que todos os trinta itens do ITC fossem explicados por uma variável latente. Para esse modelo, foi encontrado somente um índice de adequação satisfatório, o Comparative Fit Index (Tabela 1). O modelo trifatorial, que representa a teoria descrita por Beck et al. (1997), foi calculado com dez itens para cada variável latente de acordo com os estudos de Beckham et al. (1986) e Pössel (2009). Dos índices de adequação avaliados para o modelo trifatorial, tem-se o Root Mean Squared of the Residuals com escore limítrofe e o Comparative Fit Index como satisfatório. O modelo para cinco fatores foi elaborado de acordo com a proposta descrita em Anderson e Skidmore (1995) e obteve três índices de adequação satisfatórios (Root Mean Squared of the Residuals, Comparative Fit Index e Tucker-Lewis Index).

Finalmente, o modelo com seis fatores, encontrado por Pössel (2009), descreve os três fatores originais teorizados por Beck subdivididos em positivos e negativos. Os resultados para esse modelo mostraram escores satisfatórios para os três índices de adequação analisados (Root Mean Squared of the Residuals, Comparative Fit Index e Tucker-Lewis Index). Além disso, apresentou a menor razão Qui-Quadrado/Grau de liberdade e o menor Akaike Information Criterion de todos os modelos calculados.

\section{Consistência interna e validade de construto do ITC}

A partir da avaliação dos Alphas de Cronbach e correlações item-fator, foi possível obter informações sobre a consistência interna e validade de construto do ITC. O primeiro é uma medida da confiabilidade dos escores do teste relacionada à sua homogeneidade (Urbina, 2007) e o segundo relaciona-se à capacidade dos itens de representarem o construto (traço latente) a ser medido (Pasquali, 2007). Os resultados são apresentados na Tabela 2.

\section{Tabela 2. Itens divididos por fatores com respectivas correlações item-total}

Self Positivo (alpha $=0,65$ )

17 Eu me comporto tão bem quanto as outras pessoas que eu conheço.

25 Eu posso fazer bem várias coisas.

3I Eu sou uma pessoa com muitas qualidades.

0,54

33 Eu gosto de mim mesmo.

0,40

Self Negativo (alpha $=0,75$ )

5 Eu sou um fracasso.

10 Tenho estragado quase todas as relações importantes que já tive.

0,49

13 Eu não consigo fazer nada direito.

0,56

2I Eu me odeio.

0,57

29 Sou culpado por muitas coisas.

0,49

35 Eu tenho defeitos graves.

0,45 
Item

Mundo Positivo (alpha $=0,64)$

3 A maioria das pessoas são amigáveis e prestativas.

8 As pessoas que eu conheço me ajudam quando eu preciso.

12 Minhas atividades diárias são divertidas e gratificantes.

0,28

20 As pessoas importantes na minha vida são prestativas e me apoiam.

0,48

24 Eu tenho um(a) companheiro(a) ou amigos que são calorosos e prestativos.

0,44

Mundo Negativo (alpha $=0,6 \mathrm{I}$ )

180 mundo é um lugar muito hostil.

23 Acontecem muitas coisas ruins comigo.

27 Minha família não se importa com o que acontece comigo.

30 Não importa o que eu faça, as outras pessoas tornam as coisas que eu quero mais difíceis.

34 Eu estou enfrentando muitas dificuldades.

0,33

Futuro Positivo (alpha $=0,68)$

6 Eu gosto de pensar sobre as coisas boas que terei pela frente.

9 Espero que as coisas deem certo para mim a partir de agora.

0,49

I I O futuro reserva muita emoção para mim.

0,40

28 As coisas vão funcionar bem para mim no futuro.

0,46

36 Espero estar contente e satisfeito com o passar dos anos.

0,40

Futuro Negativo (alpha $=0,64$ )

15 Não resta nada em minha vida para desejar.

16 Meus problemas ou preocupações atuais estarão sempre presentes, de uma forma ou de outra.

19 Não há nenhuma razão para eu estar esperançoso quanto ao futuro.

0,49

26 Meu futuro é simplesmente terrível demais para se pensar.

0,44

32 Não há nada para desejar nos próximos anos.

0,50

Nota: Os itens I (Eu tenho muitos talentos e habilidades.), 2 (Meu trabalho [trabalho doméstico, trabalho escolar, tarefas diárias] é desagradável.), 4 (Nada parece dar certo para mim.), 7 (Eu faço o meu trabalho [emprego, escola, trabalho doméstico] adequadamente.), I4 (As pessoas gostam de mim.) e 22 (Eu vou superar meus problemas.) são itens que não pertencem à estrutura fatorial.

Fonte: Elaborada pelos autores.

Os escores encontrados pelo Alpha de Cronbach variaram de 0,61 (visão negativa do mundo) a 0,75 (visão negativa de si mesmo). De modo geral, consideram-se como aceitáveis valores maiores que 0,70 (Hora, Monteiro, \& Arica, 2010); contudo, nas ciências sociais, um valor de 0,60 pode ser tomado como aceitável (Maroco \& Garcia-Marques, 2006). As correlações dos itens com o total de cada fator mostraram sete escores inferiores a 0,40 , e três deles estão no fator visão negativa do mundo. 


\section{Inventário da Tríade Cognitiva, sexo e idade}

Comparações entre os fatores, o escore total do ITC e o sexo dos participantes mostraram uma diferença significativa na visão negativa do futuro, tendo as mulheres escores superiores aos dos homens $(t=2,95, p<0,01)$. Não foram encontradas associações superiores a 0,40 entre a idade e os escores totais no ITC. Em relação aos fatores, a idade correlacionou-se de maneira significativa com as visões positiva de si mesmo $(r=0,22, p<$ $0,001)$, negativa de si mesmo $(r=0,21, p<0,001)$ e de futuro negativo $(r=0,19, p<0,001)$. $\mathrm{Na}$ Tabela 3 estão descritas as médias, desvio padrão e quartis dos escores do ITC.

Tabela 3. Média (desvio padrão) e quartis dos fatores do Inventário da Tríade Cognitiva

\begin{tabular}{ccccc}
\hline Fator ITC & Média (DP) & Percentil 25 & Mediana & Percentil 75 \\
\hline Self Positivo & $22,24(3,62)$ & 20,00 & 23,00 & 25,00 \\
\hline Self Negativo & $33,23(5,86)$ & 30,00 & 34,00 & 38,00 \\
\hline Mundo Positivo & $26,64(4,20)$ & 24,00 & 27,00 & 30,00 \\
\hline Mundo Negativo & $24,84(4,90)$ & 22,00 & 25,00 & 28,00 \\
\hline Futuro Positivo & $30,27(4,00)$ & 29,00 & 31,00 & 33,00 \\
\hline Futuro Negativo & $29,82(4,18)$ & 28,00 & 30,50 & 33,00
\end{tabular}

Nota: Em razão da direção da escala Likert, todos os itens oriundos dos fatores positivos foram invertidos. Assim, valores elevados indicam altos índices de pensamentos positivos (para os fatores positivos) e altos índices de pensamentos negativos (para os fatores negativos).

\section{Discussão}

O objetivo deste estudo foi verificar a estrutura fatorial e avaliar algumas propriedades psicométricas do ITC, com o intuito final de obter uma medida válida para a avaliação desse importante elemento do modelo cognitivo da depressão em adultos (Beck et al., 1997).

Os resultados encontrados nas análises fatoriais confirmatórias apontam para a melhor adequação do modelo de seis fatores, em detrimento da estrutura de um, três e cinco fatores. Esse achado vai ao encontro da hipótese elaborada por Anderson e Skidmore (1995) e dos resultados encontrados por Pössel (2009), nos quais os trinta itens do CTI se dividiram entre as versões positivas e negativas da tríade cognitiva (visão de self, mundo e futuro). Algumas hipóteses podem ser elaboradas na tentativa de compreender esses resultados.

A divisão da tríade cognitiva em seis fatores pode ser tomada como uma evidência da independência entre os aspectos positivos e negativos do construto. Assim, pode-se afirmar que as visões positivas e negativas da tríade não representam polos extremos de um 
mesmo continuum, o que pode implicar em papéis diferenciados desses pensamentos na compreensão da depressão e outras psicopatologias. É importante destacar que a independência entre os aspectos positivos e negativos já foi evidenciada em outros estudos que investigavam o papel de construtos cognitivos no funcionamento psicológico, como os pensamentos automáticos (Garamoni, Reynolds, Thase, Frank, \& Fasiczka, 1992; Hogendoorn, Wolter, Vervoort, Prins, Boer, Kooij, \& Haan, 2010; Ingram \& Wisnicki, 1988).

Ao mesmo tempo, é possível que o resultado encontrado possa estar relacionado à composição da amostra do estudo. O modelo cognitivo da depressão foi desenvolvido por Beck a partir de estudos empíricos e avaliações clínicas de indivíduos deprimidos, que apresentavam visões negativas sobre si mesmos, os outros e sobre o futuro. Também a partir de investigações com sujeitos deprimidos foi empreendida a construção do Cognitive Triad Inventory (Beckham et al., 1986). Em contrapartida, tanto o estudo de Anderson e Skidmore (1995) quanto o de Pössel (2009) e o presente estudo foram conduzidos com indivíduos sem diagnósticos clínicos. Dessa forma, pode-se pensar que, em indivíduos deprimidos, a tríade cognitiva negativa apresenta uma configuração predominantemente negativa, mascarando a qualidade positiva e negativa do construto. Enquanto isso, nos indivíduos não deprimidos, a independência entre os pensamentos positivos e negativos se evidencia.

As análises de consistência interna do ITC apontaram para índices aceitáveis a adequados, com Alphas de Cronbach variando de 0,61 a 0,75. O número reduzido de itens em cada fator pode explicar os alfas encontrados, no entanto, esses índices podem ser considerados adequados quando comparados aos obtidos no estudo de Pössel (alfas entre 0,47 e 0,86 ), que investigou a mesma estrutura em alemães. A maior parte dos itens (30) apresentaram coeficientes de correlação item-fator adequados, acima de 0,40 (Hair, Black, Babin, Anderson, \& Tatham, 2006). Entretanto, sete itens apresentaram correlações entre 0,28 e 0,33 , sendo cinco deles relacionados às visões positiva e negativa das outras pessoas. Cabe destacar que não foram feitas análises semelhantes com os itens da versão original da escala (Beckham et al., 1986), e que no estudo com a versão alemã também foram encontrados vários itens com correlações item-total abaixo de 0,40 (Pössel, 2009).

Entre os resultados verificou-se que quanto maior a idade dos participantes maiores foram seus escores nos fatores futuro e self negativo e self positivo, achado que pode ser relacionado à ideia de que as crenças centrais se desenvolvem ao longo da infância e da adolescência, mas vão se consolidando ao longo dos anos à medida que são ativadas e confirmadas (Beck et al., 1997). Entretanto, apesar de estatisticamente significativas, as correlações entre os três fatores e a idade foram de baixa magnitude. Em relação ao sexo, encontrou-se que as mulheres apresentaram uma visão de futuro mais negativa do que os homens, resultado que pode ser relacionado a evidências acumuladas na literatura acerca da maior vulnerabilidade cognitiva à depressão em mulheres (Hyde, Mezulis, \& Abramson, 2008). Contudo, como não foram encontradas diferenças significativas nos demais fatores, o resultado deve ser tomado com cautela. 


\section{Considerações finais}

A adaptação do Inventário da Tríade Cognitiva apresentou uma estrutura fatorial compatível com a descrita por Pössel (2009) e, consequentemente, diferente do modelo teórico concebido por Beck et al. (1997). Esse resultado aponta para a necessidade em avaliar, separadamente, crenças e pensamentos negativos e positivos.

O presente estudo não teve uma amostra aleatorizada, nem mesmo representantes da população em condições clínicas, ou de outras regiões brasileiras, e tais fatos limitam a generalização dos resultados encontrados. A investigação da tríade cognitiva em grupos clínicos possibilitaria a verificação da estrutura fatorial e propriedades psicométricas do instrumento nessa população, e, consequentemente, a adequação do instrumento enquanto ferramenta de rastreio diagnóstico e de avaliação de mudanças ao longo da intervenção psicoterápica. Outra limitação importante do estudo relaciona-se aos índices de consistência interna, que variaram de aceitáveis $(\alpha<0,60)$ a adequados $(\alpha>0,70)$, levando à necessidade de interpretar os resultados com cautela.

Pode-se concluir que o presente estudo se insere em um panorama de crescente preocupação com a verificação empírica das qualidades psicométricas dos instrumentos utilizados em pesquisas dentro da perspectiva cognitiva. Os resultados obtidos apresentam implicações teóricas e práticas, na medida em que sinalizam a possibilidade de separação entre as tríades cognitivas negativa e positiva. Novas pesquisas utilizando amostras mais abrangentes e grupos clínicos poderão contribuir com a verificação de evidências de validade do instrumento, além de propiciar a verificação do papel dessas cognições na compreensão de transtornos psicopatológicos, como a depressão, e no bem-estar de indivíduos adultos.

\section{Referências}

Akaike, H. (1974). A new look at the statistical model identification. IEEE Transactions on Automatic Control, 19(6), 716-723.

Anderson, K. W., \& Skidmore, J. R. (1995). Empirical analysis of factors in depressive cognition: the Cognitive Triad Inventory. Journal of Clinical Psychology, 51(5), 603-609.

Beck, A. T., Rush, A. J., Shaw, B. F., \& Emery, G. (1997). Terapia Cognitiva da Depressão. Porto Alegre: Artmed.

Beck, A. T., \& Alford, B. A. (2011). Depressão: causas e tratamento. Porto Alegre: Artmed.

Beckham, E. E., Leber, W. R., Wastkins, J. R., Boyer, J. L., \& Cook, J. B. (1986). Development of an instrument to measure Beck's cognitive triad: The Cognitive Triad Inventory. Journal of Consulting and Clinical Psychology, 54(4), 566-567. 
Dobson, K. S., \& Dozois, D. J. A. (2006). Fundamentos históricos e filosóficos das terapias cognitivo-comportamentais. In K. S. Dobson (Ed.). Manual de terapias cognitivo-comportamentais (pp. 17-41). Porto Alegre: Artmed.

Esbensen, A. J., \& Benson, B. A. (2007). An evaluation of Beck's cognitive theory of depression in adults with intellectual disability. Journal of Intellectual Disability Research, 51(1), 14-24.

Garamoni, G. L., Reynolds, C. F., Thase, M. E., Frank, E., \& Fasiczka, A. (1992). Shifts in affective balance during cognitive therapy of major depression. Journal of Consulting and Clinical Psychology, 60(2), 260-266.

Greening, L., Stoppelbein, L., Dhossche, D., \& Martin, W. (2005). Psychometric evaluation of a measure of Beck's Negative Cognitive Triad for Youth: Applications for African-American and Caucasian adolescents. Depression and Anxiety, 21(4), 161-169. DOI: 10.1002/da.20073.

Haaga D. A. F., Dyck M. J., \& Ernst, D. (1991). Empirical status of cognitive theory of depression. Psychological Bulletin, 110(2), 215-236.

Hair, J. F., Black, W. C., Babin, B. J, Anderson, R. E., \& Tatham, R. L. (2006). Análise multivariada de dados (5a ed). Porto Alegre: Artmed.

Hogendoorn, S. M, Wolter, L. H., Vervoort, L., Prins, P. J. M., Boer, F., Kooij, E., \& Haan, E. (2010). Measuring negative and positive thoughts in children: An adaptation of the Children's Automatic Thoughts Scale (CATS). Cognitive Therapy Research, 34(5), 467-478. DOI: 10.1007/s10608-010-9306-2.

Hora, H. R. M., Monteiro, G. T. R., \& Arica, J. (2010). Confiabilidade em questionários para qualidade: Um estudo com o coeficiente Alfa de Cronbach. Produto \& Produção, 11(2), 85-103.

Hyde, J. S., Mezulis, A. H., \& Abramson, L. Y. (2008). The ABCs of Depression: integrating affective, biological, and cognitive models to explain the emergence of gender differences in depression. Psychological Review, 115(2), 291-313.

Ingram, R. E., \& Wisnicki, K. S. (1988). Assessment of Positive Automatic Cognition. Journal of Consulting and Clinical Psychology, 56(6), 898-902.

Jacobs, L., \& Joseph, S. (1997). Notes and shorter communications: Cognitive Triad Inventory and its association with symptoms of depression and anxiety in adolescents. Personality and Individual Differences, 22(5), 169-170.

Maroco, J., \& Garcia-Marques, T. (2006). Qual a fiabilidade do teste Alfa de Cronbach? Questões antigas e soluções modernas? Laboratório de Psicologia, 4(1), 65-90.

Mclntosh, C. N., \& Fischer, D. G. (2000). Beck's Cognitive Triad: one versus three factors. Canadian Journal of Behavioural Science, 32(3), 153-157. 
Neufeld, C. B., \& Cavenage, C. C. (2010). Conceitualização cognitiva de caso: uma proposta de sistematização a partir da prática clínica e da formação de terapeutas cognitivo-comportamentais. Revista Brasileira de Terapias Cognitivas, $6(2), 3-35$.

Pasquali, L. (2007). Validade dos testes psicológicos: Será possível reencontrar o caminho? Psicologia: Teoria e Pesquisa, 23(Edição Especial), 99-107. DOI: 10.1590/ S0102-37722007000500019.

Pössel, P. (2009). Cognitive Triad Inventory (CTI): Psychometric properties and factor structure of the German translation. J. Behav. Ther. \& Exp. Psychiat, 40(2), 240-247. DOI: 10.1016/j.jbtep.2008.12.001.

Pössel, P., \& Knopf, K. (2008). An experimental test of the maintenance and vulnerability hypothesis of depression in consideration of the cognitive hierarchy. Depression and anxiety, 25(9), E47-E55.

Śliwerski, A. (2014). Psychometric properties of the Polish version of the Cognitive Triad Inventory (CTI) - preliminary study. Archives of Psychiatry and Psychotherapy, 16(1), 47-54. DOI: 10.12740/APP/21445.

Urbina, S. (2007). Fundamentos da testagem psicológica. (C. Dornelles, Trad.). Porto Alegre: Artmed.

Submissão: 30.5 .2014

Aceitação: 19.11.2015 